\title{
MAGNETOELASTIC AND OPTICAL STUDIES OF LOW TEMPERATURE MAGNETIC RELAXATION IN RARE-EARTH ORTHOALUMINATES WITH ISING IONS
}

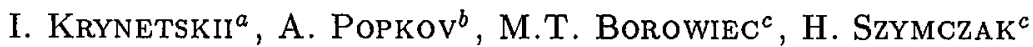 \\ AND T. ZAYARNYUK ${ }^{c}$ \\ ${ }^{a}$ Moscow State University, 119899, Vorob'evu Goru, Moscow, Russia \\ ${ }^{b}$ State Research Institute for Physical Problems \\ 103460, Zelenograd, Moscow, Russia \\ 'Institute of Physics, Polish Academy of Sciences \\ Al. Lotników 32/46, 02-668 Warsaw, Poland
}

The new class of magnetic materials with low temperature magnetic relaxation was studied. It was evidenced that the crystal $\mathrm{DyAlO}_{3}$ with antiferromagnetically ordered high anisotropy rare-earth ions showed an exponential magnetic relaxation. For the first time low temperature magnetic relaxation was investigated by means of magnetostriction and rare-earth optical absorption spectra instead of standard magnetization measurements. The possible mesoscopic nature of tunneling process was suggested.

PACS numbers: $75.40 . \mathrm{Gb}, 75.50$.Ee

Magnetic relaxation phenomena with quantum saturation at helium temperatures were studied for many years in amorphous and crystal states of bulk and film magnetics [1]. The metastable states were created usually as an effect of magnetic reversal field with amplitude being less than coercivity of the sample. The basic mechanisms of magnetic relaxation in this case were thermal and quantum creep of domain walls [2]. The quantum tunneling of domain walls should reveal itself at higher temperatures in antiferromagnets than it does in ferromagnets [3]. But using the magnetic field for metastable states creating in pure antiferromagnets leads to some problems because of the compensation of the Zeeman interaction. In our work the metastable states in antiferromagnet $\mathrm{DyAlO}_{3}$ were created by the thermal excitation. For a crystal immersed in liquid helium at the temperature of superfluid transition the helium heat conductivity changes very sharply. Due to the jump of heat removal at passing bath temperature through the $\lambda$-point at temperature increasing, the thermal shock may cause an excitation of the sample [4]. 
We suggest here a new method of metastable state relaxation study, i.e., measurement of magnetostriction at metamagnetic phase transition in rare-earth subsystem. Magnetostriction curves were measured by the use of tensometric dilatometer (relative sensitivity better than $5 \times 10^{-7}$ ) at magnetic fields up to $4.3 \mathrm{~T}$ in temperature range from $1.57 \mathrm{~K}$ to $4.2 \mathrm{~K}$. Magnetic field was directed along the $a$-axis inducing the metamagnetic phase transition $A_{y} G_{x} \Rightarrow C_{y} F_{x}$. The metamagnetic transition was accompanied by the magnetostriction anomaly which precisely displayed the rare-earth subsystem state.

The experiment was performed according to the following scheme. At the first stage, magnetostriction curves were measured at lowering sample tempera-

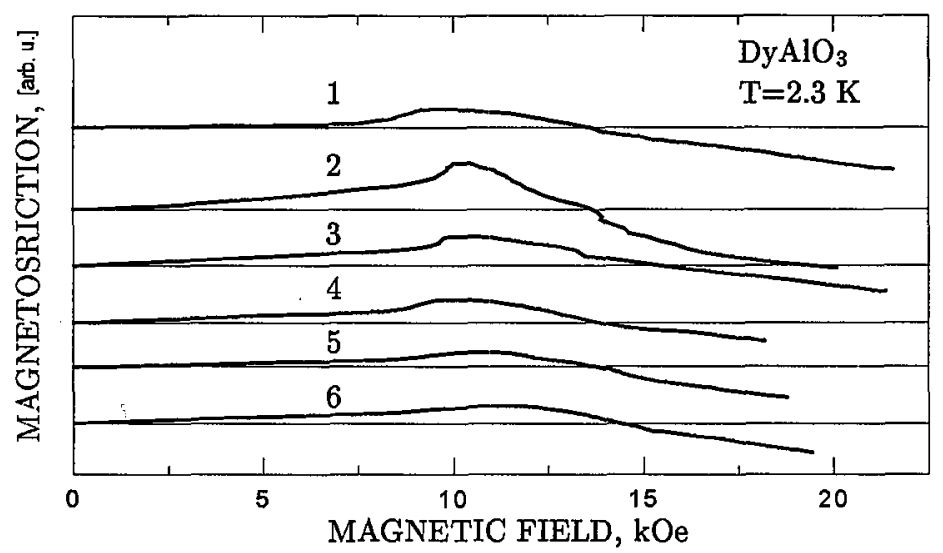

Fig. 1. The magnetostriction curves of single crystal $\mathrm{DyAlO}_{3}$ along $a$-axis at $T=2.3 \mathrm{~K}$ (curve 1 - equilibrium state of the sample; curves 2-6 - relaxation of the metastable state: $2-t=0 \mathrm{~s}, 3-280 \mathrm{~s}, 4-1120 \mathrm{~s}, 5-1680 \mathrm{~s}, 6-2240 \mathrm{~s})$.

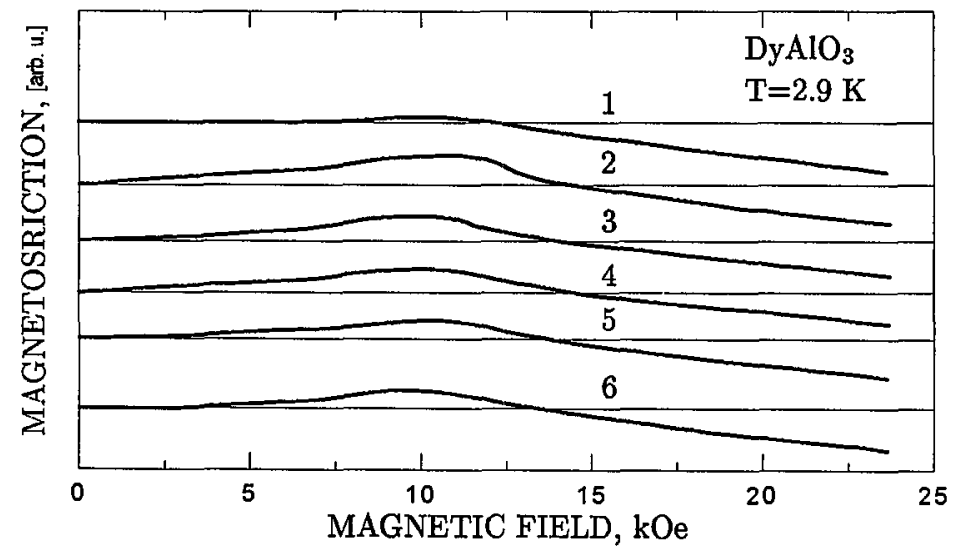

Fig. 2. The magnetostriction curves of single crystal $\mathrm{DyAlO}_{3}$ along $a$-axis at $T=2.9 \mathrm{~K}$ (curve 1 - equilibrium state of the sample; curves $2-6$ - relaxation of the metastable state: $2-t=0 \mathrm{~s}, 3-840 \mathrm{~s}, 4-1400 \mathrm{~s}, 5-3080 \mathrm{~s}, 6-3920 \mathrm{~s})$. 
ture from $4.2 \mathrm{~K}$ to $1.57 \mathrm{~K}$ (equilibrium state of the sample). As an example, Fig. 1 and Fig. 2 show magnetostriction curves measured at $T=2.3 \mathrm{~K}$ and $T=2.9 \mathrm{~K}$, respectively. All curves had pronounced anomalies caused by metamagnetic transitions. Curves marked by 1 in these figures correspond to the equilibrium state of the sample. On increasing helium bath temperature from the lowest temperature equal to $1.57 \mathrm{~K}$ the second stage of experiment started. At the $\lambda$-point temperature the sample was expressed very shortly to the heat shock due to the change of the conditions from isothermal to adiabatic ones, and magnetic subsystem of $\mathrm{DyAlO}_{3}$ was excited. Then the sample temperature was stabilized at some fixed temperature, for example equal to $2.3 \mathrm{~K}$, and successive measurements of magnetostriction curves were performed.

Further data treatment involved calculation, for each curve, of the ratio of magnetostriction anomaly (at metastable state) to the appropriate anomaly in equilibrium state. Figure 3 displayed the dependence of the logarithm of the ratio versus measurement time for the fixed temperatures. These lines allowed to determine the relaxation rates for $T=2.3 \mathrm{~K}\left(\Gamma=1.24 \times 10^{-4} \mathrm{~s}^{-1}\right)$ and for $T=2.9 \mathrm{~K}\left(\Gamma=1.53 \times 10^{-4} \mathrm{~s}^{-1}\right)$. This relaxation rate is of the same order of magnitude as that obtained for $\mathrm{TbFeO}_{3}[1]$.

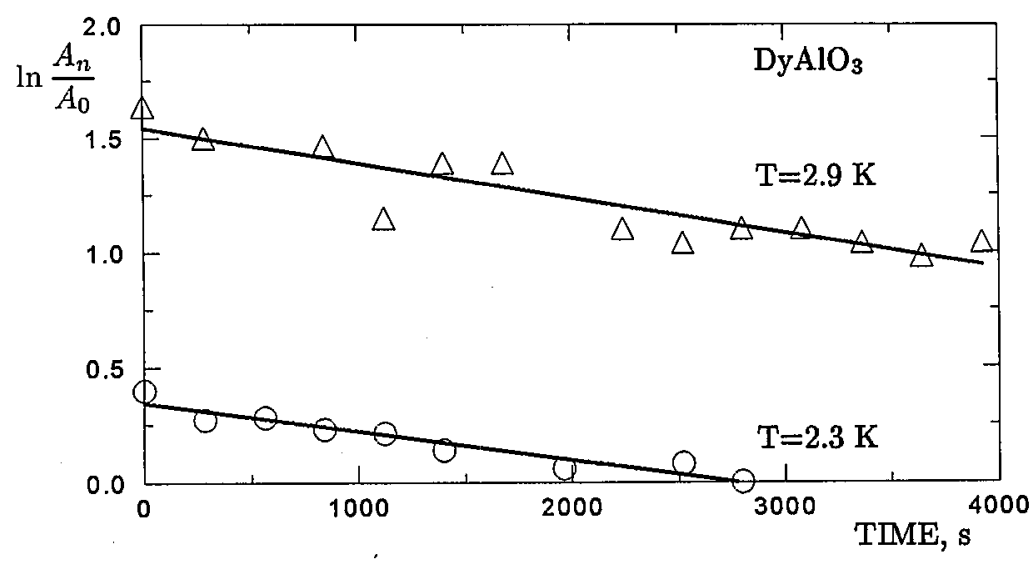

Fig. 3. Time dependence of hyperbolic logarithm of the ratio of magnetostriction anomaly at metamagnetic transition at metastable state $\left(A_{n}\right)$ to appropriate value at equilibrium state $\left(A_{0}\right)$ for $T=2.3 \mathrm{~K}$ and $T=2.9 \mathrm{~K}$.

We also studied the low temperature magnetic relaxation in $\mathrm{DyAlO}_{3}$ by means of the optical absorption spectroscopy. The transition ${ }^{6} H_{15 / 2} \Rightarrow{ }^{5} F_{3 / 2}$ was measured at some fixed temperatures before and after the application of thermal shock. The data taken at $T=2.3 \mathrm{~K}$ showed that the relative frequency shift in metastable state evaluated as $10^{-4}$ displayed the relaxation behavior.

The possible mechanisms of thermal and quantum relaxation of magnetic subsystem of the studied high anisotropy rare-earth antiferromagnet may be related to the creation of metastable antiferromagnetic phases relaxing via domain wall shift. The twofold degenerated antiferromagnetic phases result from mini- 
mization of thermodynamic potential [5]. The energies of these phases are given by

$$
\begin{aligned}
& E\left(A_{y} G_{x}\right)=\frac{1}{2}\left(-\lambda_{11}+\lambda_{12}+\lambda_{13}+\lambda_{14}\right) m^{2}, \\
& E\left(A_{x} G_{y}\right)=\frac{1}{2}\left(-\lambda_{11}+\lambda_{12}-\lambda_{13}-\lambda_{14}\right) m^{2},
\end{aligned}
$$

where $\lambda_{i j}$ are exchange parameters, and $m$ is magnetization. One of these phases is metastable and will transform into the stable phase through thermal or quantum mechanism of relaxation due to the energy pressure caused by difference of their energy density,

$$
\Delta E=E\left(A_{y} G_{x}\right)-E\left(A_{x} G_{y}\right)=\left(\lambda_{13}+\lambda_{14}\right) m^{2} .
$$

Since rare-earth Kramer's ions in $\mathrm{DyAlO}_{3}$ are not ideal Ising-like there was finite anisotropy of $g$-factor causing distribution of local symmetry of crystal field so quantum tunneling was not forbidden in this system (the quantum spin tunneling in non-Kramer's Ising ions system was considered in [6]). The evaluation of possible ions number participating in individual acts of wall phase transformation gave the values between ten and hundred. Therefore considered possible process of quantum tunneling in $\mathrm{DyAlO}_{3}$ was of mesoscopic nature in this case.

In conclusion, the exponential metastable magnetic states relaxation in rare-earth orthoaluminates with the Ising ions was found. The magnetostriction at metamagnetic phase transitions in rare-earth subsystem and the rare-earth absorption spectra were proposed as a new method of study of this phenomenon.

This work was supported by RFBR (grant 98-02-16469) and Russian Education Ministry (grant 97-0-7.3-157).

\section{References}

[1] X.X. Zhang, J. Tehada, A. Roig, O. Nikolov, E. Molins, J. Magn. Magn. Mater. 137, L235 (1994).

[2] V.V. Dobrovitski, A.K. Zvezdin, Zh. Exp. Teor. Fiz. 109, 14209 (1996).

[3] V.Yu. Golushev, A.F. Popkov, Europhys. Lett. 29, 327 (1995).

[4] N.P. Kolmakova, I.B. Krynetskii, E.V. Sinitsin, in: Proc. First Int. Meet. Magnetoelastic Effects and Applications, Naples (Italy) 1993, Ed. L. Lanotte, Elsevier, 1993, p. 297.

[5] A.K. Zvezdin, V.M. Matveev, A.A. Mukhin, A.I. Popov, Rare-Earth Ions in Magneto-ordered Crystals, Nauka, Moscow 1985 (in Russian).

[6] A.K. Zvezdin, A.F. Popkov, Pisma Zh. Exp. Teor. Fiz. 57, 548 (1993). 